\title{
Peculiar X-ray features in bright early-type galaxies
}

\author{
Ginevra Trinchieri \\ Osservatorio Astronomico di Brera, Milano, Italy
}

\section{Introduction}

IC 1262 and NGC 6159 have been selected for high resolution observations because their X-ray luminosity observed in the ROSAT All Sky Survey (Voges 1992) was higher than that observed from other normal, early type galaxies. The HRI observations confirm both the identification of the X-ray sources with the two galaxies (see Fig. 1; point sources in the field, clearly associated with optical counterparts, further ensure that the absolute pointing is correct), and the very high luminosities. Moreover, they indicate very extended emission, out to $\geq 7^{\prime}$ and $\geq 4^{\prime}$ radius ( $\geq 400$ and $\geq 220 \mathrm{kpc}$ at the galaxies' distances), respectively, with a relatively regular morphology (although not azimuthally symmetric at large radii), and a smoothly decreasing surface brightness profile. At the center of IC 1262, however, several peculiar features are present (see Fig. 3 and later discussion).
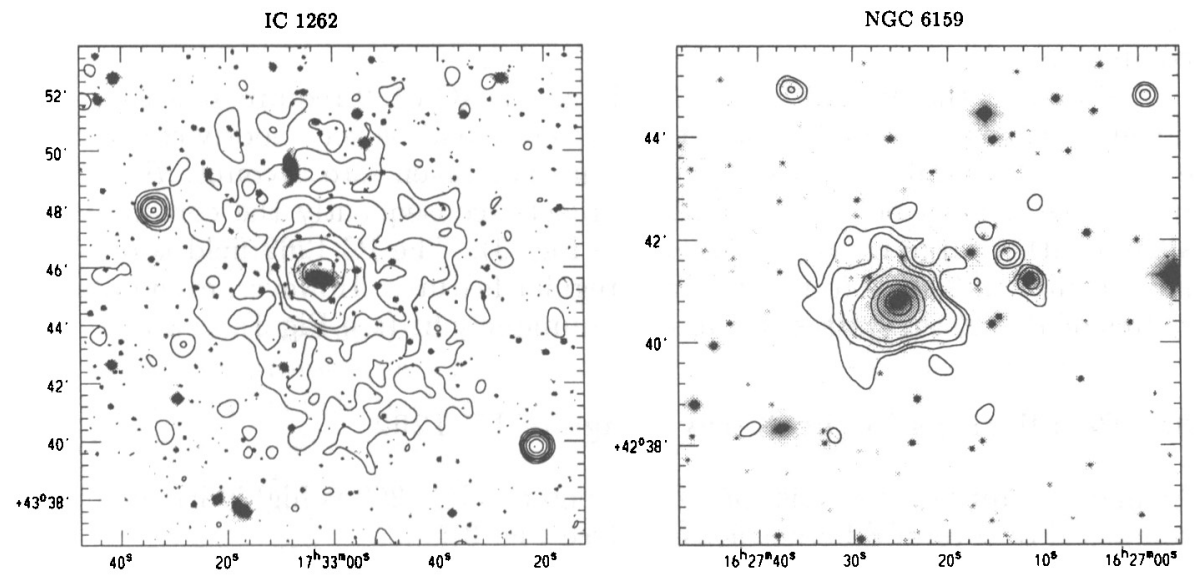

Figure 1. Isointensity contour plot of the X-ray emission in IC 1262 and NGC 6159 superposed on the DSS image. The IC 1262 HRI data have been smoothed with a Gaussian function of $15^{\prime \prime} \sigma$; the NGC 6159 HRI data with a $10^{\prime \prime} \sigma$ function.

The optical evidence for a group of galaxies around IC 1262 (Wegner et al. 1996) helps to explain the extended morphology, large $\mathrm{L}_{X}$ and large $\mathrm{L}_{\mathrm{X}} / \mathrm{L}_{\mathrm{B}}$, otherwise very peculiar for an early type galaxy. The emission around NGC 6159 
is also most likely due to a group, as suggested by the presence of other similarly bright galaxies around it, although spectroscopic confirmation is still needed.

\section{Group emission}

The bulk of the emission from these two objects can be explained as due to a group of galaxies around the two central objects, with X-ray properties comparable to those of closer, better studied groups (Burns et al. 1996; Mulchaey et al 1996; Ponman et al 1996; and this conference):

- no gas temperature can be measured with the HRI data, but a temperature of 1-2 keV and small low energy absorption can be inferred from the X-ray colors derived from the RASS data, on the assumption of a plasma spectrum.

- total X-ray $\left(0.1-2 \mathrm{keV}\right.$ ) luminosities are $\mathrm{L}_{x} \sim 4.4 \times 10^{43} \mathrm{erg} \mathrm{s}^{-1}$ (IC 1262, $D=206 \mathrm{Mpc}$ ), and $7.5 \times 10^{42} \mathrm{erg} \mathrm{s}^{-1}$ (NGC 6159, D=188 Mpc).

- average gas density and mass, derived under the assumption of spherical geometry, are $\mathrm{n}_{e} \sim 3 \times 10^{-4}$ and $\mathrm{M}_{\text {gas }} \sim 3 \times 10^{12} M_{\odot}-7 \times 10^{11} M_{\odot}$, for IC 1272 and NGC 6159 , respectively.

The X-ray radial profiles of groups and clusters have been parameterized by a "King-type" model, characterized by a core radius $r_{c}$ and an index $\beta$ for the large scale distribution. Mulchaey \& Zabludoff (1998) recently noticed that two components are required in most nearby groups: one centered on the central elliptical, with typical extent of $20-40 h^{-1} \mathrm{kpc}$, probably related to the interstellar medium of the galaxy, and a second one detected out to much larger radii, more closely related to the intragroup medium. $\beta \sim 0.8-1$ for both components.

Similarly, the data of both IC 1262 and NGC 6159 require at least 2 components. However, we find that the parameter space is very poorly constrained, even when the azimuthal asymmetries are not taken into account: $\beta=0.5$ or $\beta=1$ could both be used for either objects; consequently the core radii also span a rather large range in values (see Fig. 3). The IC 1262 field is of course more complicated due to the central irregular features. However, even outside a radius of $1^{\prime}-2^{\prime}$ the data still cannot be uniquely fit (see Fig 3 ).

\section{Peculiarities in the $\mathrm{X}$-ray image of IC $\mathbf{1 2 6 2}$}

At high resolution, the emission in the center of IC 1262 is highly irregular, and composed of several different features (see Fig. 3):

- a narrow, long arc-like feature. Its X-ray luminosity, above the average emission outside of it in the innermost $1^{\prime}$ radius region, is $\mathrm{L}_{X} \sim 4 \times 10^{42}$ $\mathrm{erg} \mathrm{s}^{-1}$. Although the peak is displaced from the galaxy, this feature is probably associated with it, since some additional X-ray emission from a most-likely unrelated point-like object visible to the $\mathrm{E}$ might move the peak and confuse the association. This arc represents a luminous perturbation on the more regular group emission, with a density contrast up to a factor of 2 relative to the immediate surroundings (under reasonable assumptions of the gas temperature and of the geometry of the feature) 

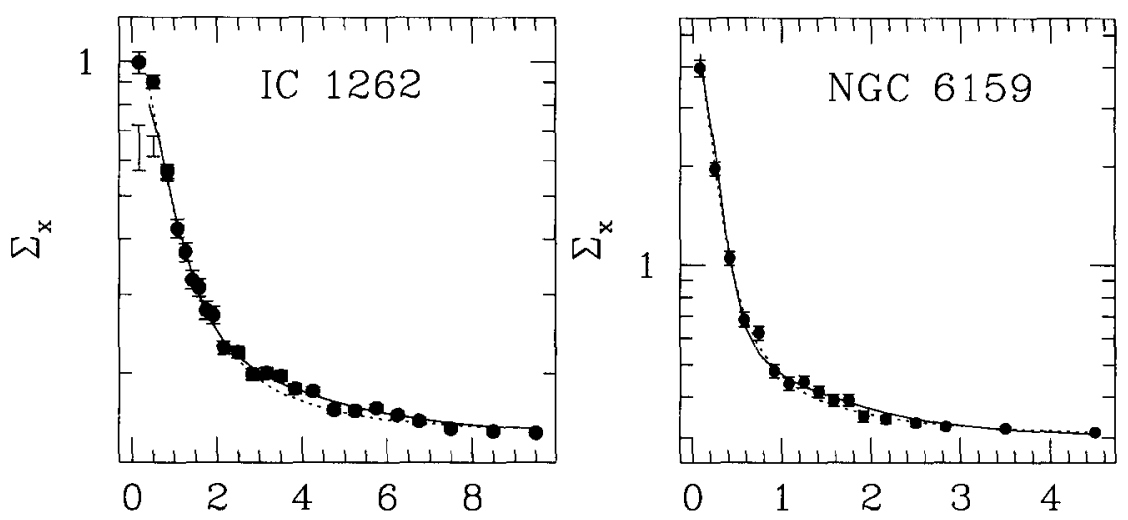

radius (arcmin)
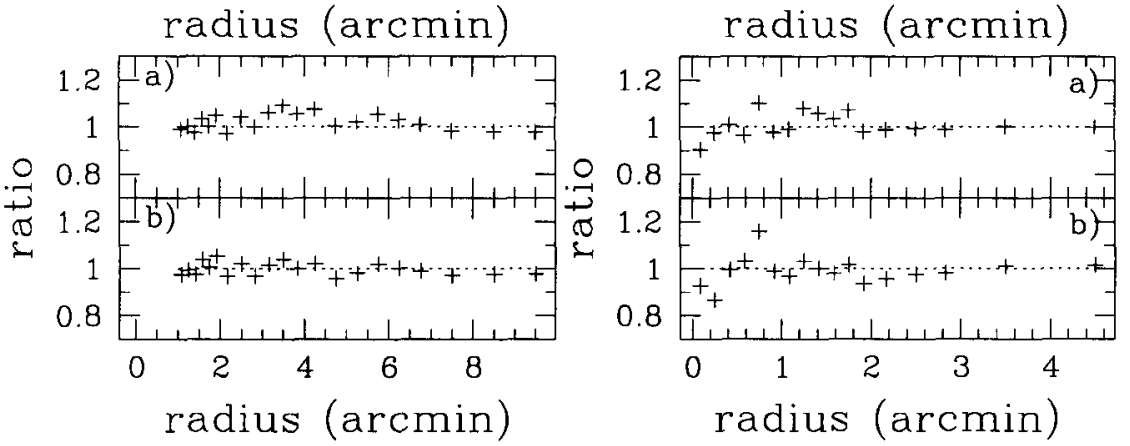

Figure 2. Comparison between the azimuthally averaged surface brightness profiles (data and errors, filled dots) and the model fits (king model(s) plus field background). IC 1262 (left): model $a: r_{c}(1)=80^{\prime \prime}$ and $\mathrm{r}_{c}(2)=400^{\prime \prime}$ and $\beta=1$ (solid line); and model $b$ : $\mathrm{r}_{c}(1)=38^{\prime \prime}$, $\beta=0.5$ (dotted line). The inner profile contains (dots) or excludes (error bars only) the peculiar central feature. NGC 6159 (right): model $a: \mathbf{r}_{c}(1)=20^{\prime \prime}, \mathbf{r}_{c}(2)=160^{\prime \prime}$ and $\beta=1$ (solid line); and model $b$ : $\mathrm{r}_{c}(1)=8^{\prime \prime}, \beta=0.5$ and $\mathrm{r}_{c}(2)=300^{\prime \prime}, \beta=1$ (dotted line). The data to model ratios are given in the bottom panels. 


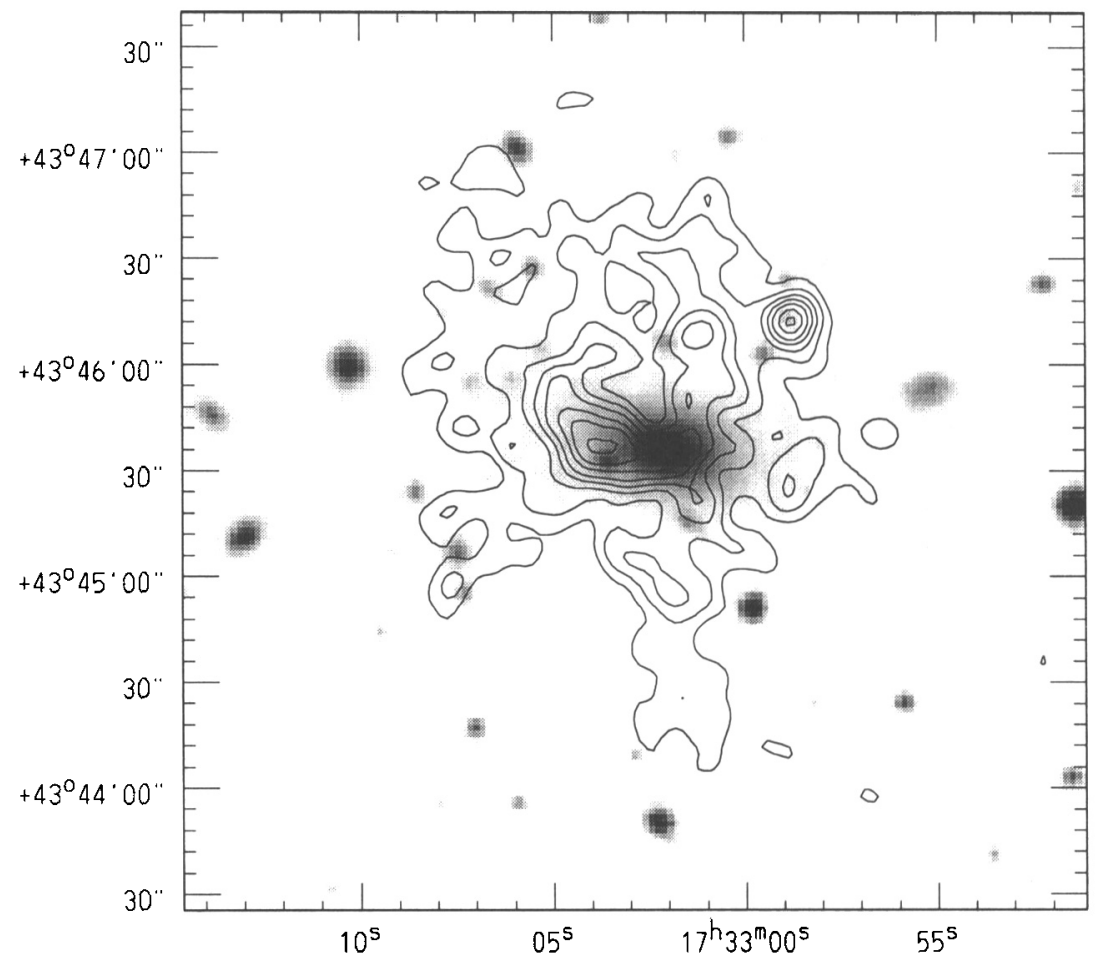

Figure 3. The central region of the HRI field of IC 1262 (isointensity contours of the original data binned to a resolution of $1 " /$ pixel and smoothed with a Gaussian function of $\sigma=4^{\prime \prime}$ ) superposed on the optical Digitized Sky Survey image. The $\mathrm{X}$-ray morphology appears complex, and it is composed of: a very peculiar arc at the center of the emission, and a tail extending to the S. The (unresolved) feature to the NW is most likely an interloper. 
- an elongation towards the south, $>1^{\prime}$ long, reminiscent of a tail. This feature also has a significant surface brightness contrast $(\geq 2 \times)$ relative to the immediate surroundings.

- a point-like source to the NW, most likely an interloper. Its X-ray-tooptical flux ratio suggests it to be a background AGN or a foreground $\mathrm{K}$-M type star.

The peculiar morphology at the center of IC 1262 is most likely due to a shock: but what could the cause of the shock be? Two explanations, not entirely satisfactory in light of our current knowledge of the system, are possible:

- A merger event: Groups and clusters are thought to be forming through subsequent accretions of new material, which most likely would produce shocks between the colliding structures (Peebles 1990). In this system, however, most of the emission appears to be in a more relaxed, regular component, suggesting that if a merger is occurring it should be a recent phenomenon, local to the center. Future data at other wavelengths might support this scenario, if evidence of a merger is discovered.

- Motion of IC 1262 in the ambient medium: The ram pressure resulting from the relative motions could very easily explain the $\mathrm{X}$-ray morphology. However, the ambient medium is hot, so that very high velocities are required to produce a shock $\left(\gg 500 \mathrm{~km} \mathrm{~s}^{-1}\right)$. Such high velocities for IC 1262 are at odds with both its classification as a cD galaxy (Saglia et al. 1997) and with the group's velocity dispersion $\left(\sim 300 \mathrm{~km} \mathrm{~s}^{-1}\right.$, although IC 1262 has indeed the lowest velocity measured, Wegner et al. 1999).

Although clearly more data is required to properly interpret the $\mathrm{X}$-ray morphology and properties of IC 1262, the high resolution observations of these two systems further point to the necessity of high quality observations of groups of galaxies to fully understand their complex dynamics and evolution processes.

\section{References}

Burns, J. O., Ledlow, M. J., Locken, C., et al. 1996, ApJL, 467, L49.

Mulchaey, J. S., et al. 1996, ApJL, 457, L19

Mulchaey, J. S. \& Zabludoff, A. I. 1998, ApJ, 496, 73

Peebles, P. J. E. 1980, The large scale structure of the Universe, Princeton Univ. Press.

Ponman T. J., et al., 1996, MNRAS, 283, 690

Saglia, R. P., Burstein, D., Baggley, G., et al. 1997, MNRAS, 292, 499

Voges, W. 1992 Proc. European IST Meeting Symposium, p. 9

Wegner, G., Colless, M., Baggley, G., et al. 1996, ApJS, 106, 1

Wegner, G., Colless, M., Saglia, R. P., et al. 1999, MNRAS, 305, 259 